\title{
Increased Prevalence of Autonomic Dysfunction due to Postural Orthostatic Tachycardia Syndrome in Patients with Eosinophilic Gastrointestinal Disorders
}

\author{
Kevin Z. Huang, Evan S. Dellon
}

Center for Esophageal

Diseases and Swallowing, Center for Gastrointestinal Biology and Disease, Division of Gastroenterology and Hepatology, University of North Carolina School of Medicine, Chapel Hill, NC, USA

\footnotetext{
Address for correspondence: Evan S. Dellon $\mathrm{CB} \# 7080$ Bioinformatics Building 130 Mason Farm Rd. $\mathrm{UNC}-\mathrm{CH}$ Chapel Hill, NC 27599-7080, USA

edellon@med.unc.edu
}

Received: 19.01.2019

Accepted: 11.02.2019

\begin{abstract}
Background \& Aims: Eosinophilic gastrointestinal disorders (EGIDs) have been linked with atopic conditions, connective tissue disorders, and autoimmunity, but the association between EGIDs and autonomic dysfunction has not been extensively described. We aimed to assess this association and determine the prevalence of autonomic dysfunction in our EGID population.

Methods: In this retrospective cohort study, we analyzed adult patients in the University of North Carolina Eosinophilic Esophagitis (EoE)/EGID database who also had a diagnosis of comorbid autonomic dysfunction (postural orthostatic tachycardia syndrome [POTS] or gastroparesis). The electronic medical record was abstracted and disease characteristics summarized.

Results: We identified seven patients who had EGID and autonomic dysfunction. All had POTS and were Caucasian, and five (71\%) were female; 2 also had gastroparesis. Onset of autonomic symptoms coincided with onset of EGID symptoms in 3 patients (43\%) or with an acute EGID flare in 3. In no cases did successful control of EGID lead to improvement of autonomic symptoms. Two subjects (29\%) had comorbid EhlersDanlos syndrome and mast cell activation syndrome. The prevalence of POTS in EGID patients was 7/560 (1.25\%), compared to an estimated $0.17 \%$ prevalence of POTS in the general population.

Conclusions: The prevalence of autonomic dysfunction was 5-10-fold higher in EGID than expected in the general population, suggesting an association between the two conditions, though the underlying mechanism is unclear. Providers should consider comorbid autonomic dysfunction in patients with EGID, particularly when symptoms persist despite treatment.
\end{abstract}

Key words: eosinophilic gastrointestinal disorder - autonomic dysfunction - postural orthostatic tachycardia syndrome.

Abbreviations: CTD: connective tissue disorder; EC: eosinophilic colitis; EDS: Ehlers Danlos Syndrome; EoE: eosinophilic esophagitis; EG: eosinophilic gastritis; EGE: eosinophilic gastroenteritis;; EGID: eosinophilic gastrointestinal diseases; GI: gastrointestinal; IBD: inflammatory bowel disease; IBS: irritable bowel syndrome; IRB: institutional review board; MCAS: mast cell activation syndrome; POTS: postural orthostatic tachycardia syndrome; TGF: transforming growth factor; TRPV1: transient receptor potential vanilloid type 1.

\section{INTRODUCTION}

Eosinophilic gastrointestinal disorders (EGID) are chronic allergen/immune-mediated disorders characterized by eosinophilic inflammation in the gastrointestinal (GI) tract and symptoms referable to the site of GI tract involvement. Eosinophilic gastrointestinal disorders include eosinophilic esophagitis (EoE), gastritis (EG), gastroenteritis (EGE), and colitis (EC). Although EGID are thought to be allergen-mediated, studies have also linked EGID with connective tissue disorders [1] and other autoimmune disorders [2]. Similarly, postural orthostatic tachycardia syndrome (POTS) has been linked to connective tissue disorders and autoimmune diseases, but the association between EGID and POTS, as well as other conditions of autonomic dysfunction, has not been extensively described.

POTS belongs to a group of disorders under the term autonomic dysfunction, pathology that stem from dysregulation and dysfunction of the autonomic nervous system. POTS is characterized by an excessive increase in heart rate in the absence of orthostatic hypotension and that is not explained 
by medications or other causes of tachycardia [3]. Diagnosis is established with: 1) an increase of $30 \mathrm{bpm}$ within 10 minutes of tilt table testing; 2) lack of hypotension, which is defined as a decrease in systolic BP $>20 \mathrm{mmHg}$ or diastolic BP $>10 \mathrm{mmHg}$; and 3) associated symptoms such as lightheadedness, palpitations, vision changes, and generalized weakness. Treatments include physical conditioning, increased fluid/ salt intake, and medicines such as propranolol, midodrine, fludrocortisone, and pyridostigmine. POTS is a final common pathway for many diseases and has been associated with Ehlers-Danlos syndrome (EDS), mast cell activation syndrome (MCAS), chronic fatigue syndrome, and autoimmune disorders $[3,4]$. Gastroparesis is another common form of autonomic dysfunction. Gastroparesis stems from dysfunctional GI motility, resulting in gastric stasis, delayed gastric emptying, and symptoms of nausea, vomiting, and abdominal pain.

Gastrointestinal symptoms and impaired gastric motility are prevalent in up to $69 \%$ of patients with POTS [5], and gastroparesis is a common culprit given the shared pathophysiology between POTS and gastroparesis. However, patients with POTS and GI symptoms have also been shown to have a higher prevalence of comorbid irritable bowel syndrome (IBS) and inflammatory bowel diseases (IBD) [6], as well as other functional GI disorders [5]. Coexisting EGID could be another explanation for GI symptoms in POTS, and we have clinically observed an association. However, this association has not been extensively described. Therefore, our aims were to identify patients with EGID and POTS, describe the association between these two entities, and assess the prevalence of autonomic dysfunction in our EGID population.

\section{METHODS}

This was a retrospective cohort study conducted at the University of North Carolina (UNC). We identified adult patients ( $>18$ years old) in the EoE/EGID database at UNC who also had a diagnosis of comorbid autonomic dysfunction (POTS or gastroparesis). The details of this database have been previously described [7-12]. In brief, pathology records and billing codes are routinely monitored to identify all patients with EGID diagnosis. Eosinophilic esophagitis was defined as per consensus guidelines $[13,14]$. As there are no consensus guidelines for EG, EGE, or EC, these have been defined as per recent recommendations for histologic thresholds for GI eosinophilia, in the presence of appropriate symptoms, and in the absence of competing secondary causes of eosinophilia [15]. Within this EoE/EGID database, we screened for cases of comorbid autonomic dysfunction (POTS and gastroparesis) via record review. Cases were then verified by identifying documentation of positive tilt-table testing and gastric emptying studies for POTS and gastroparesis, respectively.

Once all cases were identified, the electronic medical record was abstracted using a standardized case report form for data regarding their EGID and POTS, including demographic information, disease characteristics and diagnostic information, treatments, and presence of other comorbidities.

For analysis, we used descriptive statistics (proportions, means) to summarize characteristics of the study population.
We calculated the prevalence of autonomic dysfunction in EGID patients at UNC. This study was approved by the UNC IRB.

\section{RESULTS}

We identified 7 patients who had EGID and autonomic dysfunction. All had POTS, all were Caucasian, and 5 were female. There were 4 patients with EoE alone, 2 with EGE and overlapping esophageal involvement, and 1 with EGE overlapping with EC. In addition to POTS, 2 patients also had gastroparesis (Table I). The median age (in years) at diagnosis of EGID was 28, ranging from 16 to 54 .

Table I. Patient demographics, type of eosinophilic gastrointestinal disorder (EGID), autonomic dysfunction, and other comorbidities in a cohort of seven patients with comorbid EGID and autonomic dysfunction

\begin{tabular}{lcc}
\hline Patient Demographics & $\begin{array}{c}\text { Number of } \\
\text { patients }\end{array}$ & $(\%)$ \\
\hline White & 7 & $100 \%$ \\
Female & 5 & $71 \%$ \\
EGID & 4 & $57 \%$ \\
EoE & 2 & $29 \%$ \\
EoE + EGE & 1 & $14 \%$ \\
EG + EC & & \\
Autonomic Dysfunction & 7 & $100 \%$ \\
POTS & 2 & $29 \%$ \\
Gastroparesis & & \\
Comorbidities & 2 & $29 \%$ \\
Ehlers Danlos & 2 & $14 \%$ \\
Mast Cell Activation & 3 & \\
Hypothyroidism & 1 & $29 \%$ \\
Celiac Disease & & \\
\hline
\end{tabular}

POTS symptoms, from most to least common, included presyncope, palpitations, lightheadedness, dizziness, dyspnea, true syncope, diaphoresis, "black out vision", and "brain fog" (Table II). Of the six patients with esophageal involvement (with EoE alone or with another overlapping EGID), the most common symptoms were dysphagia and epigastric abdominal pain, followed by heartburn, nausea and vomiting. Of the three patients with EGE, the most common symptoms were nausea and vomiting and abdominal pain (Table III).

Onset of POTS symptoms coincided with onset of EGID symptoms in 3 patients or with an acute EGID flare in 3 patients. Other potential triggers of autonomic symptoms included pregnancy in two patients and transition to college in one patient. For POTS, 6 of the patients sought treatment with an autonomic dysfunction specialist. The most common treatments included liberalized fluid and salt intake, physical conditioning, corticosteroids (hydrocortisone, fludrocortisone) and pyridostigmine (Table II). Oral viscous budesonide, swallowed fluticasone, targeted dietary elimination, elemental diet, and/or endoscopic dilations were sufficient to control disease activity in the four patients with only EoE. The three patients with EGE (overlapping with either esophageal involvement or EC) required systemic steroids to control their 
Table II. Frequency of symptoms and attempted treatments for postural orthostatic tachycardia syndrome (POTS) in our cohort of seven patients with comorbid eosinophilic gastrointestinal disorder (EGID) and autonomic dysfunction.

\begin{tabular}{|c|c|c|}
\hline POTS Symptoms & $\begin{array}{l}\text { Number of } \\
\text { patients }\end{array}$ & $(\%)$ \\
\hline Presyncope & 5 & $71 \%$ \\
\hline Palpitations & 5 & $71 \%$ \\
\hline Lightheadedness & 3 & $43 \%$ \\
\hline Dizziness & 3 & $43 \%$ \\
\hline Dyspnea & 3 & $43 \%$ \\
\hline True syncope & 2 & $29 \%$ \\
\hline Diaphoresis & 2 & $29 \%$ \\
\hline „Black out vision” & 2 & $29 \%$ \\
\hline „Brain fog” & 2 & $29 \%$ \\
\hline Confusion & 1 & $14 \%$ \\
\hline \multicolumn{3}{|l|}{ POTS treatment } \\
\hline Liberal fluid/salt intake & 4 & $57 \%$ \\
\hline Exercise & 4 & $57 \%$ \\
\hline Fludrocortisone & 3 & $43 \%$ \\
\hline Pyridostigmine & 3 & $43 \%$ \\
\hline $\begin{array}{l}\text { Beta blockers (propranol, metoprolol, } \\
\text { carvedilol, nebivolol) }\end{array}$ & 2 & $29 \%$ \\
\hline Midodrine & 1 & $14 \%$ \\
\hline Hydrocortisone & 1 & $14 \%$ \\
\hline SSRIs (citalopram, sertraline) & 1 & $14 \%$ \\
\hline Ivabridine & 1 & $14 \%$ \\
\hline Diltiazem & 1 & $14 \%$ \\
\hline No documentation & 1 & $14 \%$ \\
\hline
\end{tabular}

EGID (Table III). In no case did the successful control of EGID lead to the improvement of autonomic symptoms. Median follow up time after EGID remission was 4 years, and ranged from 1 to 8 years.

Two patients had comorbid EDS and two patients MCAS. One patient had both EDS and MCAS. Three patients had hypothyroidism (one of whom was documented as Hashimoto's), and one had celiac disease (Table I). None of the patients studied had comorbid diabetes mellitus.

Using the UNC EoE/EGID database ( $\mathrm{n}=560$ adults at the time this study was performed), we calculated a prevalence of POTS in EGID patients as 7/560 (1.25\%), compared to the estimated previously published $0.17 \%$ prevalence of POTS in the general population $[3,16]$.

\section{DISCUSSION}

Given the inflammatory involvement of the GI tract in EGID, the variable symptoms associated with these conditions, and the fact that eosinophils can impact both motility and nerve function, it is plausible that there is an association between EGID and autonomic dysfunction. We had anecdotally observed this association during routine clinical care, but this has not been extensively evaluated in the medical literature. Our data suggest that POTS is perhaps 5-10 fold more prevalent in patients with EGID than in the general
Table III. Frequency of symptoms and successful treatments for EGID in our cohort of seven patients with comorbid eosinophilic gastrointestinal disorder (EGID) and autonomic dysfunction. Six patients had esophageal involvement (EoE alone or in combination with other EGID) and three patients had EGE with other EGID.

\begin{tabular}{|c|c|c|}
\hline & $\begin{array}{l}\text { Number of } \\
\text { patients }\end{array}$ & $(\%)$ \\
\hline \multicolumn{3}{|l|}{ EoE symptoms $(n=6)$} \\
\hline Dysphagia & 5 & $83 \%$ \\
\hline Epigastric abdominal pain & 5 & $83 \%$ \\
\hline Heartburn & 3 & $50 \%$ \\
\hline $\mathrm{N} / \mathrm{V}$ & 2 & $33 \%$ \\
\hline \multicolumn{3}{|l|}{ EoE treatments $(n=6)$} \\
\hline Elimination diet & 3 & $50 \%$ \\
\hline Oral viscous budesonide & 2 & $33 \%$ \\
\hline Flovent & 1 & $17 \%$ \\
\hline Elemental diet & 1 & $17 \%$ \\
\hline Dilation & 1 & $17 \%$ \\
\hline \multicolumn{3}{|l|}{ EGE symptoms $(\mathrm{n}=3)$} \\
\hline Nausea and vomiting & 3 & $100 \%$ \\
\hline Abdominal pain & 3 & $100 \%$ \\
\hline \multicolumn{3}{|l|}{ EGE treatments $(n=3)$} \\
\hline Systemic steroids & 3 & $100 \%$ \\
\hline Elemental diet & 1 & $33 \%$ \\
\hline Colchicine & 1 & $33 \%$ \\
\hline
\end{tabular}

population. Moreover, onset of POTS tended to coincide with onset/flares of EGID in our patients, potentially suggesting a pathogenic association between EGID and POTS. Although two of the patients in our cohort also had gastroparesis, it remains unclear whether EGID is associated with other forms of autonomic dysfunction.

Given this possible association, we tried to identify distinguishing features of our patients' EGID and POTS compared to typical presentations. Patients all had typical presentations of EGID, with EoE predominantly presenting with dysphagia, and EGE causing mostly abdominal pain, nausea and vomiting. Similarly, the most common POTS symptoms (palpitations, lightheadedness, dizziness, and dyspnea) were all observed in most of our patients. Interestingly, the demographics of this subset of patients showed a strong female predominance. Although this is consistent with non-EoE EGIDs as well as with POTS [3], EoE has a male predominance, and the majority (4/7) of our patients had EoE alone [17].

Underlying mechanisms for the association between EGID and POTS remain unclear. One possible mechanism involves the underlying inflammation in EGID directly modulating the autonomic nervous system and causing symptoms of autonomic dysfunction. Many allergy disorders manifest with symptoms that are mediated by changes in the nervous system, and mechanisms of allergen-induced neuromodulation have been previously described [18]. Previous studies have demonstrated that mast cells and eosinophils can act as neuroactive mediators at sites of allergic inflammation, sensitizing the nervous system response to antigens. This has 
been shown in EoE as well, with one study demonstrating that allergic inflammation can mediate afferent neural signals in the vagal sensory ganglia in the guinea pig model of EoE via transient receptor potential vanilloid type 1 (TRPV1) [19]. The study suggests that inflammation in the esophagus similar to that found in EoE leads to formation of neurotrophic factors that upregulate transcription of TRPV1 in neurons, thereby modulating the function of the nervous system and in particular causing visceral hypersensitivity. As this was a retrospective study and biopsies were clinically taken only of the mucosa, we were not able to evaluation eosinophil and nerve interactions. Further research is required to determine if a similar pathway is present in humans, and if a form of allergen-induced neuromodulation could underlye a mechanism in which EGID could lead to autonomic symptoms such as those in POTS. Additional investigations would also be required to understand if fibrosis related to EGE could impact on gastric function and lead to gastroparesis.

Another possibility is that a separate distinct process could be underlying or leading to the development of both EGID and POTS. Within our cohort, we identified EDS and MCAS as possibilities. Ehlers Danlos syndrome, seen in two of our patients, is a connective tissue disorder (CTD) that has an association with POTS that has been well established [2022]. Recent studies have demonstrated a higher prevalence of EoE among patients with CTD such as EDS [1], and have shown that dysregulation in transforming growth factor beta (TGF- $\beta$ ) levels and subsequent errors in signaling can underly the pathogenesis of both EGID and CTD [23]. TGF- $\beta$ may also mediate dysmotility in the esophagus, though it is unknown if this may be at play throughout the rest of the GI tract. Similarly observed in two of our patients, MCAS has been shown to be associated with hyperadrenergic POTS [24]. Abnormal mast cell release of vasoactive mediators lead to vasodilation and subsequent orthostatic intolerance. Mast cells are implicated in the pathophysiology of immune/allergy mediated disorders such as EGID, and MCAS has been linked to atopic diseases such as asthma and allergic rhinitis, with which EGID is also linked. Moreover, studies have demonstrated that some patients with EoE have elevated tryptase levels indicative of increased mast cell activation [25]. Ehlers Danlos syndrome and MCAS have been linked as well [26], raising the possibility of a unifying mechanism underlying all four syndromes (EGID, POTS, EDS, and MCAS).

An autoimmune etiology could also connect both EGID and POTS. Eosinophilic esophagitis has been associated with other autoimmune conditions such as Hashimoto's (as seen in our cohort), multiple sclerosis, and lupus [27]. POTS has been linked also with autoimmune disorders [4, 28, 29].

Alternatively, our study population could represent a coincidental overlap of symptoms, and such a small sample size is insufficiently powered to conduct a statistical analysis. Our study is also limited by its retrospective design, with data obtained from a chart review. Notably, we were unable to discern the POTS subtypes (i.e. neuropathic POTS, hyperadrenergic POTS, etc.) from a chart review alone. However, these limitations are countered by the large patient population assessed with comprehensive case finding strategies and standardized extraction of data.

\section{CONCLUSIONS}

Our data suggests that POTS is 5-10 fold more prevalent in patients with EGID than in the general population, and that EGID may be associated with POTS and gastroparesis. Patients with EGID and POTS tended to be female, but otherwise presented with typical symptoms of both disorders. POTS symptoms persisted in our patients despite EGID treatments. Although further research examining other cohorts is required to determine if this is a true association, providers should consider comorbid autonomic dysfunction in patients with EGID. Because our data suggests that autonomic symptoms are likely to persist despite treatment of EGID, providers should consider referral to an autonomic specialist for treatment of these symptoms even if EGID itself is well controlled.

Conflicts of interest: None of the authors have any potential conflicts related to this manuscript.

Authors' contributions: K.Z.H. and E.S.D: project conception, data collection, data analysis, data interpretation, manuscript preparation, critical revision, final approval. E.S.D: supervision.

Acknowledgements: This paper was supported by NIH R01 DK101856 and NIH T35 DK007386.

\section{REFERENCES}

1. Abonia JP, Wen T, Stucke EM, et al. High prevalence of eosinophilic esophagitis in patients with inherited connective tissue disorders. J Allergy Clin Immunol 2013;132:378-386. doi:10.1016/j.jaci.2013.02.030

2. Peterson K, Firszt R, Fang J, Wong J, Smith KR, Brady KA. Risk of Autoimmunity in EoE and Families: A Population-Based Cohort Study. Am J Gastroenterol 2016;111:926-932. doi:10.1038/ajg.2016.185

3. Garland EM, Celedonio JE, Raj SR. Postural Tachycardia Syndrome: Beyond Orthostatic Intolerance. Curr Neurol Neurosci Rep 2015;15:60. doi:10.1007/s11910-015-0583-8

4. Blitshteyn S. Autoimmune markers and autoimmune disorders in patients with postural tachycardia syndrome (POTS). Lupus 2015;24:1364-1369. doi:10.1177/0961203315587566

5. Mehr SE, Barbul A, Shibao CA. Gastrointestinal symptoms in postural tachycardia syndrome: a systematic review. Clin Auton Res 2018;28:411421. doi:10.1007/s10286-018-0519-x

6. Wang LB, Culbertson CJ, Deb A, Morgenshtern K, Huang H, Hohler AD. Gastrointestinal dysfunction in postural tachycardia syndrome. J Neurol Sci 2015;359:193-196. doi:10.1016/j.jns.2015.10.052

7. Sperry SL, Woosley JT, Shaheen NJ, Dellon ES. Influence of race and gender on the presentation of eosinophilic esophagitis. Am J Gastroenterol 2012;107:215-221. doi:10.1038/ajg.2011.342

8. Runge TM, Eluri S, Cotton CC, et al. Outcomes of Esophageal Dilation in Eosinophilic Esophagitis: Safety, Efficacy, and Persistence of the Fibrostenotic Phenotype. Am J Gastroenterol 2016;111:206-213. doi:10.1038/ajg.2015.399

9. Wolf WA, Jerath MR, Sperry SL, Shaheen NJ, Dellon ES. Dietary Elimination Therapy Is an Effective Option for Adults With Eosinophilic Esophagitis. Clin Gastroenterol Hepatol 2014;12:1272-1279. doi:10.1016/j.cgh.2013.12.034

10. Reed C, Woosley JT, Dellon ES. Clinical characteristics, treatment outcomes, and resource utilization in children and adults with 
eosinophilic gastroenteritis. Dig Liver Dis 2015;47:197-201. doi:10.1016/j.dld.2014.11.009

11. Dellon ES, Fritchie KJ, Rubinas TC, Woosley JT, Shaheen NJ. Interand intraobserver reliability and validation of a new method for determination of eosinophil counts in patients with esophageal eosinophilia. Dig Dis Sci 2010;55:1940-1949. doi:10.1007/s10620-0091005-Z

12. Eluri S, Runge TM, Hansen J, et al. Diminishing Effectiveness of LongTerm Maintenance Topical Steroid Therapy in PPI Non-Responsive Eosinophilic Esophagitis. Clin Transl Gastroenterol 2017;8:e97. doi:10.1038/ctg.2017.27

13. Liacouras CA, Furuta GT, Hirano I, et al. Eosinophilic esophagitis: Updated consensus recommendations for children and adults. J Allergy Clin Immunol 2011;128:3-20.e6. doi:10.1016/j.jaci.2011.02.040

14. Dellon ES, Gonsalves N, Hirano I, et al. ACG Clinical Guideline: Evidence based approach to the diagnosis and management of esophageal eosinophilia and eosinophilic esophagitis (EoE). Am J Gastroenterol 2013;108:679-692. doi:10.1038/ajg.2013.71

15. Collins MH. Histopathologic features of eosinophilic esophagitis and eosinophilic gastrointestinal diseases. Gastroenterol Clin North Am 2014;43:257-268. doi:10.1016/j.gtc.2014.02.007

16. Robertson D. The epidemic of orthostatic tachycardia and orthostatic intolerance. Am J Med Sci 1999;317:75-77.

17. Dellon ES, Jensen ET, Martin CF, Shaheen NJ, Kappelman MD. Prevalence of eosinophilic esophagitis in the United States. Clin Gastroenterol Hepatol 2014;12:589-596.e1. doi:10.1016/j. cgh.2013.09.008

18. Undem BJ, Taylor-Clark T. Mechanisms underlying the neuronal-based symptoms of allergy. J Allergy Clin Immunol 2014;133:1521-1534. doi:10.1016/j.jaci.2013.11.027

19. Liu Z, Hu Y, Yu X, et al. Allergen challenge sensitizes TRPA1 in vagal sensory neurons and afferent $\mathrm{C}$-fiber subtypes in guinea pig esophagus. Am J Physiol Gastrointest Liver Physiol 2015;308:G482-G488. doi:10.1152/ajpgi.00374.2014
20. Wallman D, Weinberg J, Hohler AD. Ehlers-Danlos Syndrome and Postural Tachycardia Syndrome: a relationship study. J Neurol Sci 2014;340:99-102. doi:10.1016/j.jns.2014.03.002

21. Hakim A, O'Callaghan C, De Wandele I, Stiles L, Pocinki A, Rowe P. Cardiovascular autonomic dysfunction in Ehlers-Danlos syndromeHypermobile type. Am J Med Genet C Semin Med Genet 2017;175:168174. doi:10.1002/ajmg.c.31543

22. Gazit Y, Nahir AM, Grahame R, Jacob G. Dysautonomia in the joint hypermobility syndrome. Am J Med 2003;115:33-40. doi:10.1016/ S0002-9343(03)00235-3

23. Frischmeyer-Guerrerio PA, Guerrerio AL, Oswald G, et al. TGFbeta receptor mutations impose a strong predisposition for human allergic disease. Sci Transl Med 2013;5:195ra94. doi:10.1126/ scitranslmed.3006448

24. Shibao C, Arzubiaga C, Roberts LJ 2nd, et al. Hyperadrenergic postural tachycardia syndrome in mast cell activation disorders. Hypertension 2005;45:385-390. doi:10.1161/01.HYP.0000158259.68614.40

25. Kutty GR, Downs-Kelly E, Crispin HT, Peterson KA. Elevated Tryptase in EoE Is an Independent Phenomenon Associated with ExtraEsophageal Symptoms. Dig Dis Sci 2019;64:152-157. doi:10.1007/ s10620-018-5298-7

26. Seneviratne SL, Maitland A, Afrin L. Mast cell disorders in EhlersDanlos syndrome. Am J Med Genet C Semin Med Genet 2017;175:226236. doi:10.1002/ajmg.c.31555

27. Jensen, E.T., et al., Su1852 High prevalence of co-existing autoimmune conditions among patients with eosinophilic esophagitis. Gastroenterology, 2013;144 (5 Suppl 1):S-491. doi:10.1016/S00165085(13)61817-X

28. Li H, Yu X, Liles C, et al. Autoimmune basis for postural tachycardia syndrome. J Am Heart Assoc 2014;3:e000755. doi:10.1161/ JAHA.113.000755

29. Watari M, Nakane S, Mukaino A, et al. Autoimmune postural orthostatic tachycardia syndrome. Ann Clin Transl Neurol 2018;5:486-492. doi:10.1002/acn3.524 